\title{
Reliability and validity of an FFQ for South American children and adolescents from the SAYCARE study
}

\author{
Tara Rendo-Urteaga ${ }^{1,2, *}$, (0, Luisa Saravia ${ }^{2,3}$, Tatiana Sadalla Collese ${ }^{1,2}$,
} Julia María Monsalve-Alvarez ${ }^{4}$, Laura Inés González-Zapata ${ }^{4} \odot$, Florencia Tello ${ }^{5}$, Beatriz Martínez-Oliván ${ }^{2}$, Francisco Leonardo Torres-Leal ${ }^{6}$, Luis A Moreno ${ }^{7}$, Augusto César Ferreira De Moraes ${ }^{1,8}$ and Heráclito Barbosa Carvalho'

IYCARE (Youth/Child cArdiovascular Risk and Environmental) Research Group, School of Medicine, University of São Paulo, São Paulo, SP, Brazil: ${ }^{2}$ Growth, Exercise, NUtrition and Development (GENUD) Research Group, Faculty of Health Science (EUCS), Instituto Agroalimentario de Aragón (IA2), University of Zaragoza-CITA, Zaragoza, Spain:

${ }^{3}$ School of Nutrition, University of La República, Montevideo, Uruguay: ${ }^{4}$ School of Nutrition and Dietetics, University of Antioquia, Medellin, Colombia: ${ }^{5}$ Escuela de Nutrición, Facultad de Medicina, Universidad de Buenos Aires, Buenos

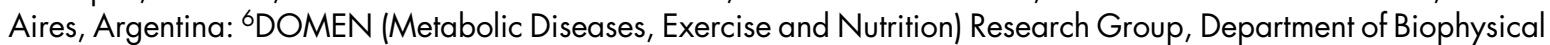
and Physiology, Federal University of Piauí, Teresina, Brazil: ${ }^{7}$ Instituto de Investigación Sanitaria de Aragón (IIS Aragón) y Centro de Investigación Biomédica en Red de Fisiopatología de la Obesidad y Nutrición (CIBEROBN), Instituto de Salud Carlos III, Madrid, Spain: ${ }^{8}$ Department of Epidemiology, Bloomberg School of Public Health, Johns Hopkins University, Baltimore, MD, USA

Submitted 10 September 2018: Final revision received 19 March 2019: Accepted 7 May 2019: First published online 12 September 2019

\begin{abstract}
Objective: The purpose of this study was to analyse the reliability and validity of a semi-quantitative FFQ to assess food group consumption in South American children and adolescents.

Design: The SAYCARE (South American Youth/Child cARdiovascular and Environmental) study is an observational, multicentre, feasibility study performed in a sample of 3- to 18-year-old children and adolescents attending private and public schools from six South American countries. Participants answered the FFQ twice with a two-week interval and three 24-h dietary recalls. Intraclass and Spearman's correlations, weighted Cohen's kappa ( $\kappa w)$, percentage of agreement and energy-adjusted Pearson's correlation coefficients were calculated.

Setting: Seven cities in South America (Buenos Aires, Lima, Medelin, Montevideo, Santiago, Sao Paulo and Teresina).

Subjects: A sample of 200 children and 244 adolescents for reliability analyses and 252 children and 244 adolescents for validity analyses were included.

Results: Depending on the food group, for children and adolescents, reliability analyses resulted in Spearman's coefficients from 0.47 to 0.73 , intraclass correlation coefficients from 0.66 to $0.99, \kappa w$ coefficients from 0.35 to 0.63 , and percentage of agreement between 72.75 and $83.52 \%$. In the same way, validity analyses resulted in Spearman's coefficients from $0 \cdot 17$ to $0 \cdot 37$, energy-adjusted Pearson's coefficients from 0.17 to $0.61, \kappa w$ coefficients from 0.09 to 0.24 , and percentages of agreement between 45.79 and $67.06 \%$.

Conclusion: The SAYCARE FFQ achieved reasonable reliability and slight-moderate validity for almost all food groups intakes. Accordingly, it can be used for the purpose of ranking the intake of individuals within a population.
\end{abstract}

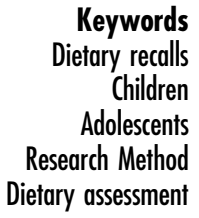

Keywords Children

Adolescents Dietary assessment
Dietary intake is an important determinant of nutritional status and health in children and adolescents. An accurate assessment of food intake is essential for monitoring nutritional status, identifying diet-related behaviours in youth, and conducting epidemiological and clinical research to optimize current and future health ${ }^{(1)}$.

The most common dietary methodologies used to assess dietary intake are diet history, food diaries (dietary 
records), food recalls and $\mathrm{FFQ}^{(2)}$. FFQ are the most frequently used tool applied in large cross-sectional and cohort studies and nutritional related studies ${ }^{(3,4)}$, as they capture usual dietary intake over longer periods of time compared with other methods and can be collected from a large number of participants in a relatively short time frame ${ }^{(2,5)}$. FFQ are practical, easy-to-administer and inexpensive tools that question the frequency of intake from a previously defined list of foods over a specific period of time. This method can be self- or interview administered and is a potentially valuable tool to assess habitual dietary intake among children and adolescents $^{(3,5)}$. Despite the suggestion that overestimation may occur when the FFQ had many food items ${ }^{(4)}$, these questionnaires are appropriate tools to explore factors associated with changes in the dietary patterns of a population and have been demonstrated to be useful tools for investigating the association between diet and metabolic and $\mathrm{CVD}^{(6)}$.

There are additional challenges concerning self-reporting while assessing food intake in youths through the use of food questionnaires. Children may have limitations regarding an adequately developed concept of time, memory and attention span and may lack knowledge of food names, preparation, and portion size ${ }^{(3,7)}$. In adolescents, issues of motivation, body image and difficulty reporting portion sizes can hinder the willingness to report ${ }^{(7,8)}$. Although the FFQ reproducibility and validity have been assessed in children and adolescents as wells as in adults ${ }^{(8,9)}$, measuring energy and food intake at an early age is particularly challenging because there are few valid tools for the younger population ${ }^{(3)}$. Due to these limitations, it is essential to select the appropriate method in accordance with the study design, target population and outcome of interest ${ }^{(10)}$.

Several multicentre studies regarding lifestyle and cardiovascular health in European children and adolescents have used standardized reliable and valid methods to assess food intake ${ }^{(11-13)}$. To allow comparisons between countries, this appears to be the best strategy. Validation studies are necessary to indicate the effect of measurement error and to prevent incorrect estimations ${ }^{(4)}$. Currently, there are no validated tools to assess food intake among South American children and adolescents. Given the increasing prevalence of childhood obesity worldwide, and also in South America, it seems necessary to have a tool that can be applied in epidemiological studies to help understand the origin of the non-communicable diseases related to lifestyle in children and adolescents. Therefore, in spite of the great challenge that implies working with a young population, from different countries with different culture and language, among other aspects, this study aimed to analyse the reliability and validity of an FFQ to assess food group consumption in this population.

\section{Methods}

\section{Study design}

The SAYCARE (South American Youth/Child cARdiovascular and Environmental) study is a observational, multicentre, feasibility study performed in a convenience sample of 3- to 18-year-old children and adolescents attending private and public schools from the following seven cities in six South American countries: Buenos Aires (Argentina), Lima (Peru), Medelin (Colombia), Montevideo (Uruguay), Santiago (Chile) and Sao Paulo and Teresina (Brazil). Random sampling was conducted by using student lists and each sex was represented by $50 \%$ of participants. All parents/guardians signed an informed consent form, and a signed assent form was obtained from children/ adolescents to indicate their approval to participate in the study. The exclusion criteria were inability to complete the questionnaires and pregnancy. Data collection occurred between 2015 and 2016. A detailed description of the SAYCARE study has been published elsewhere ${ }^{(14)}$. The study was performed in accordance with the ethical standards of the Declaration of Helsinki and was approved by the Research Ethics Committee of each city involved.

\section{Data collection}

Participants were enrolled in the SAYCARE study for approximately 4 weeks. All SAYCARE questionnaires were answered by the children's caregivers (3-10 years), while adolescents (11-18 years) answered it themselves. Participants answered the FFQ twice and three 24-h dietary recalls (24HDR) (Fig. 1). Caregivers or adolescents received verbal and written instructions on how to complete these questionnaires. The instructions incorporated standardized examples and a coloured food photo booklet, which contained photographs of commonly consumed food (including country-specific foods) and their standard portion sizes, to facilitate accurate recordings ${ }^{(15)}$. Data for this study were analysed in March 2017. Total energy intake was calculated from FFQ1 data using the United States Department of Agriculture (USDA) ${ }^{(16)}$ food composition databases and a food composition database ${ }^{(17)}$ from each SAYCARE country when local foods were not found in the USDA database.

\section{Reliability and validity of the FFQ}

We developed a semi-quantitative FFQ for children and adolescents from South America, as described elsewhere $^{(15)}$. Depending on the city and their country-specific foods included in the FFQ, the questionnaire had different numbers of items: for Buenos Aires, the FFQ had a total of 63 items, Lima had a total of 61 items, 63 items for Medelin, 59 items for Montevideo, 57 items for Santiago, 67 items for Sao Paulo, and 69 items for Teresina. To assess FFQ reliability, all participants answered the FFQ twice with a twoweek interval between assessments (FFQ1 and FFQ2) 


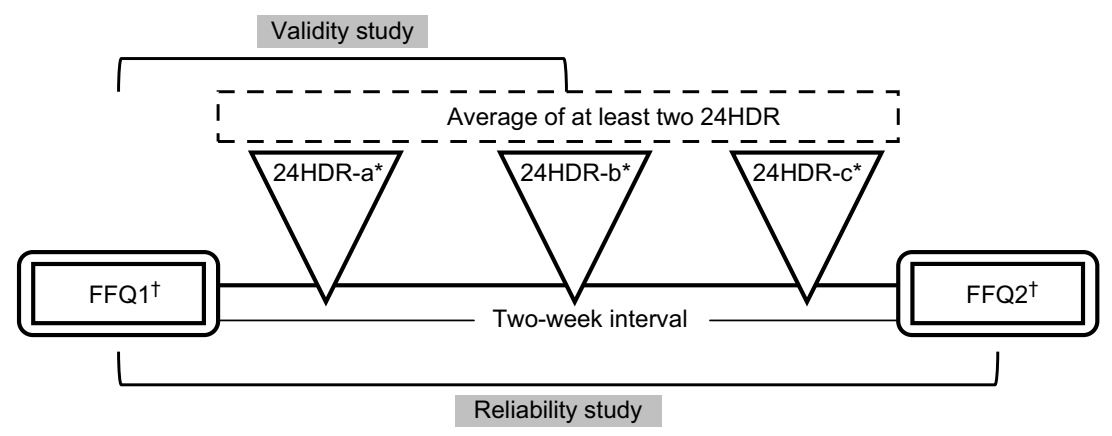

Fig. 1 Design of the reliability and validity analyses among children and adolescents of the SAYCARE study. ${ }^{*}$ First $24 \mathrm{HR}$ with a

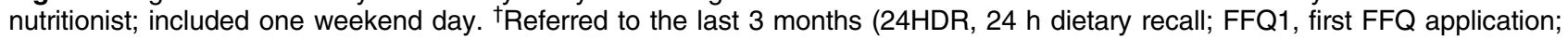
FFQ2, second FFQ application)

(Fig. 1). Data from the FFQ1 and FFQ2 were entered in the SAYCARE platform ${ }^{(14)}$. This is a secured web-based system that facilitates data collection, entry, management and analysis of the SAYCARE data ${ }^{(14)}$. Our FFQ asked the frequency and portion size of each food item ${ }^{(15)}$. Each frequency was transformed into a daily frequency with each portion recorded in grams or millilitres. Therefore, portion sizes were multiplied by their respective frequencies to obtain total intake of each food item in grams or millilitres per day. Subsequently, food items were summed into food groups according to the $\mathrm{FAO}^{(18)}$ as follows: (i) Cereals; (ii) Tubers; (iii) Vegetables; (iv) Fruits; (v) Oils; (vi) Meat and Derivatives, Fish and Eggs; (vii) Milk and Dairy products; (viii) Legumes; (ix) Beverages; (x) Sugar products; and (xi) Miscellaneous. For adolescents, we assessed the ninth group, 'Beverages', with and without alcoholic drinks as a different group.

Additionally, in three non-consecutive 24HDR (two weekdays and one weekend day), caregivers and adolescents had to describe all foods consumed during the previous day, qualitatively and quantitatively using household measures (Fig. 1). This questionnaire was structured into six daily eating occasions. Children caregivers and adolescents answered the first 24HDR in school with a trained dietitian, and the second and third 24HDR were answered at home with the support of the food booklet used for the FFQ. In Buenos Aires and Lima, caregivers answered the three $24 \mathrm{HDR}$ at home (except in special situations such as non-literate caregivers who needed help to respond to the questionnaires and they did it in school with the help of a trained dietitian). Data from 24HDR were entered into computer-based food software developed to evaluate energy and nutrient intake from Ibero-American food consumption surveys. The food composition databases included in it are the Spanish Food Composition Database (Bedca); the National Nutrient Database for Standard Reference Release (USDA); and the IberoAmerican Foods Database. Moreover, some typical Brazilian foods were inserted into this software, considering the Brazilian Food Composition Table (TACO). Food items from this software were exported to a Microsoft Excel spreadsheet and checked by a trained nutritionist. Recorded food items in the $24 \mathrm{HDR}$ were matched to the items as defined by the FFQ. An example of this was 'bread' or 'rice' from the $24 \mathrm{HDR}$. This was assigned to the corresponding item in the FFQ and then to the FAO group 'cereals'. Each ingredient in a composite dish was assigned with a proportion size of meal and then allocated to the respective food group. For instance, a ham and cheese sandwich' was proportionally recorded in the three corresponding food groups in FFQ as follows: 'cereals', 'meat and derivatives' and 'milk and dairy products'. Data obtained from at least two $24 \mathrm{HDR}$, in grams (or millilitres per day for beverages), were summed, and the mean was calculated to provide average daily intakes. Finally, food items from the $24 \mathrm{HDR}$ were summed into food groups according to the FAO as previously described to enable a direct comparison between FFQ1 and the 24HDR (validity analysis).

For the reliability analysis, sample size was calculated using $k=0 \cdot 70, \alpha=5 \%$ and $\beta=80 \%$. For the validity analysis, $k=0 \cdot 40, \alpha=5 \%$ and $\beta=80 \%$ were used. The estimated sample size was 146 participants for the reliability analysis and 40 for the validity analysis. Anticipating potential sample losses, a $25 \%$ greater sample size was recruited in each city, which was equally divided by sex and public/private schools. These sample sizes were considered appropriate according to our sample size calculations and according to Willett (2009) for studies validating dietary intake tools ${ }^{(2)}$.

\section{Statistical analysis}

All statistical analyses were performed using the statistical software package Stata version 14.0, they included all countries and were performed by age group. The Shapiro-Wilk test was used to determine variable distribution. Continuous non-normally distributed variables are presented in median and 95\% CI. Categorical variables are shown as proportions (\%). To assess differences between reported intakes between questionnaires 
Table 1 Main characteristics of the SAYCARE population for reliability and validity analyses

\begin{tabular}{|c|c|c|c|c|c|c|c|c|}
\hline & \multicolumn{4}{|c|}{ Reliability analyses (FFQ1 v. FFQ2) } & \multicolumn{4}{|c|}{ Validity analyses (FFQ1 v. 24HDR) } \\
\hline & \multicolumn{2}{|c|}{$\begin{array}{c}\text { Children (3-10 years) } \\
\text { ( } n \text { 200) }\end{array}$} & \multicolumn{2}{|c|}{$\begin{array}{c}\text { Adolescents (11-18 } \\
\text { years) ( } n \text { 244) }\end{array}$} & \multicolumn{2}{|c|}{$\begin{array}{c}\text { Children (3-10 years) } \\
\text { ( } n \text { 252) }\end{array}$} & \multicolumn{2}{|c|}{$\begin{array}{l}\text { Adolescents (11-18 } \\
\text { years) }(n \text { 244) }\end{array}$} \\
\hline & Median & $95 \% \mathrm{Cl}$ & Median & $95 \% \mathrm{Cl}$ & Median & $95 \% \mathrm{Cl}$ & Median & $95 \% \mathrm{Cl}$ \\
\hline Age (years) & $5 \cdot 90$ & $6 \cdot 03,6 \cdot 62$ & $15 \cdot 00$ & $14 \cdot 28,14 \cdot 82$ & $5 \cdot 65$ & $5 \cdot 88,6 \cdot 37$ & $14 \cdot 80$ & $14 \cdot 28,14.83$ \\
\hline \multicolumn{9}{|l|}{ Gender (\%) } \\
\hline Female & $50 \cdot 50$ & & 49.59 & & 53.17 & & 52.05 & \\
\hline Male & $49 \cdot 50$ & & $50 \cdot 41$ & & $46 \cdot 83$ & & 47.95 & \\
\hline Weight (kg) & 21.67 & $22 \cdot 45,24 \cdot 76$ & 54.00 & $54.33,57.58$ & $21 \cdot 22$ & $22 \cdot 01,23 \cdot 88$ & 53.45 & $53.58,56.83$ \\
\hline Height (m) & $1 \cdot 15$ & $1.14,1.18$ & 1.58 & $1.58,1.60$ & 1.15 & $1.13,1 \cdot 17$ & 1.58 & $1.57,1.60$ \\
\hline BMI $\left(\mathrm{kg} / \mathrm{m}^{2}\right)$ & $16 \cdot 4$ & $16 \cdot 56,17 \cdot 29$ & 21.49 & $21 \cdot 39,22 \cdot 44$ & $16 \cdot 26$ & $16 \cdot 56,17 \cdot 30$ & 20.99 & $21 \cdot 21,22 \cdot 29$ \\
\hline \multicolumn{9}{|l|}{ School type (\%) } \\
\hline Public & 61.50 & & 38.93 & & $59 \cdot 13$ & & 44.76 & \\
\hline Private & 38.50 & & 61.07 & & 40.87 & & 55.33 & \\
\hline \multicolumn{9}{|l|}{ Maternal education level (\%) } \\
\hline Without education & 0 & & 1.64 & & 0 & & 1.30 & \\
\hline Low education & $10 \cdot 87$ & & 7.38 & & 11.25 & & $10 \cdot 39$ & \\
\hline Low secondary education & $8 \cdot 70$ & & 4.92 & & $7 \cdot 50$ & & $5 \cdot 84$ & \\
\hline High secondary education & $10 \cdot 87$ & & 22.95 & & 13.75 & & 24.68 & \\
\hline Technical education & $10 \cdot 87$ & & 13.93 & & $12 \cdot 50$ & & $10 \cdot 39$ & \\
\hline University degree & 58.70 & & $49 \cdot 18$ & & 55.00 & & 47.40 & \\
\hline
\end{tabular}

Continuous non-normally distributed variables were presented in median and $95 \% \mathrm{Cl}$ and categorical variables were presented as proportion (\%).

Reliability analyses: First $v$. second FFQ application (FFQ1 v. FFQ2).

Validity analyses: First FFQ application (FFQ1) v. average of at least two 24-h dietary recalls (24HDR).

(FFQ1, FFQ2 and 24HDR), the Wilcoxon test was performed. We used the Spearman rank correlation coefficients (Spearman's rho) for continuous nonparametric data to assess FFQ reliability and validity. Pearson's correlation coefficients were then calculated and presented before and after to adjust for total energy intake ${ }^{(19)}$, for validity analysis. For all food group intakes, data were logtransformed $\left(\log _{10}\right)$ prior to multilevel regression analyses to improve normality. Random or fixed effect models for each food group were used according to the Housman test. The multilevel analyses were performed with 'centre' as the contextual variable and total energy intake as the individual variable. Furthermore, for reliability, intraclass correlation coefficients (ICC) were calculated since this analysis takes account of between- and within- subject variability in responses. Values of ICC less than 0.40 indicate poor reliability, values between 0.41 and 0.75 indicate fair to good reliability, and values greater than 0.75 indicate excellent reliability. Finally, tertiles of intake for each food group were calculated, and weighted Cohen's kappa ( $\kappa w)$ coefficients were used (for categorical data) to evaluate the agreement in ranking. Values of $\kappa w$ over 0.80 indicate very good agreement, values between 0.61 and 0.80 indicate substantial (good) agreement, values from 0.41 to 0.60 indicate moderate agreement, values from 0.21 to 0.40 indicate fair agreement and values between 0 and 0.20 indicate slight (poor) agreement $^{(20)}$. Classification into the same or an adjacent tertile was subsequently calculated. Our sample size was estimated to guarantee statistical significance for correlation coefficients $r>0 \cdot 20^{(2)}$. The significance level for all tests were set at $P<0.05$.

\section{Results}

A total of 345 children and 357 adolescents answered the FFQ1, and 202 children and 247 adolescents answered the FFQ2. In addition, 86 children responded to two $24 \mathrm{HDR}$ and 323 children answered three 24HDR (being a total of 409 children who answered at least two 24HDR); in addition, 60 adolescents answered two 24HDR and 241 adolescents answered the three 24HDR (being a total of 301 adolescents who answered at least two 24HDR) (data not shown). Descriptive analyses are presented in Table 1 divided by reliability/validity analyses and age group. A total of 200 children (50.5\% female; median age 5.9 years) and 244 adolescents ( $49.6 \%$ female; median age 15.0 years) were included in the reliability analyses, and a total of 252 children $(53.2 \%$ female; median age 5.6 years) and 244 adolescents ( $52.0 \%$ female; median age 14.8 years) were included in the validity analyses.

Daily reported intakes from the FFQ and reliability results are shown in Table 2 . When comparing intakes reported in both FFQ, FFQ1 provided higher intake estimates for cereals, vegetables and beverages in children and for cereals, milk and dairy products and both groups of beverages in adolescents. We found acceptable reliability in the food groups according to Spearman's $\rho$ coefficients (ranging from 0.49 to 0.73 in children and from 0.47 to 0.67 in adolescents) and good to excellent reliability (ranging from 0.87 to 0.99 in children and from 0.66 to 0.99 in adolescents) according to ICC. According to classification into tertiles of intake, the percentage of agreement (children/adolescents classified in the same or adjacent tertile) ranged from 70.75 to $83.52 \%$ in children, and from 


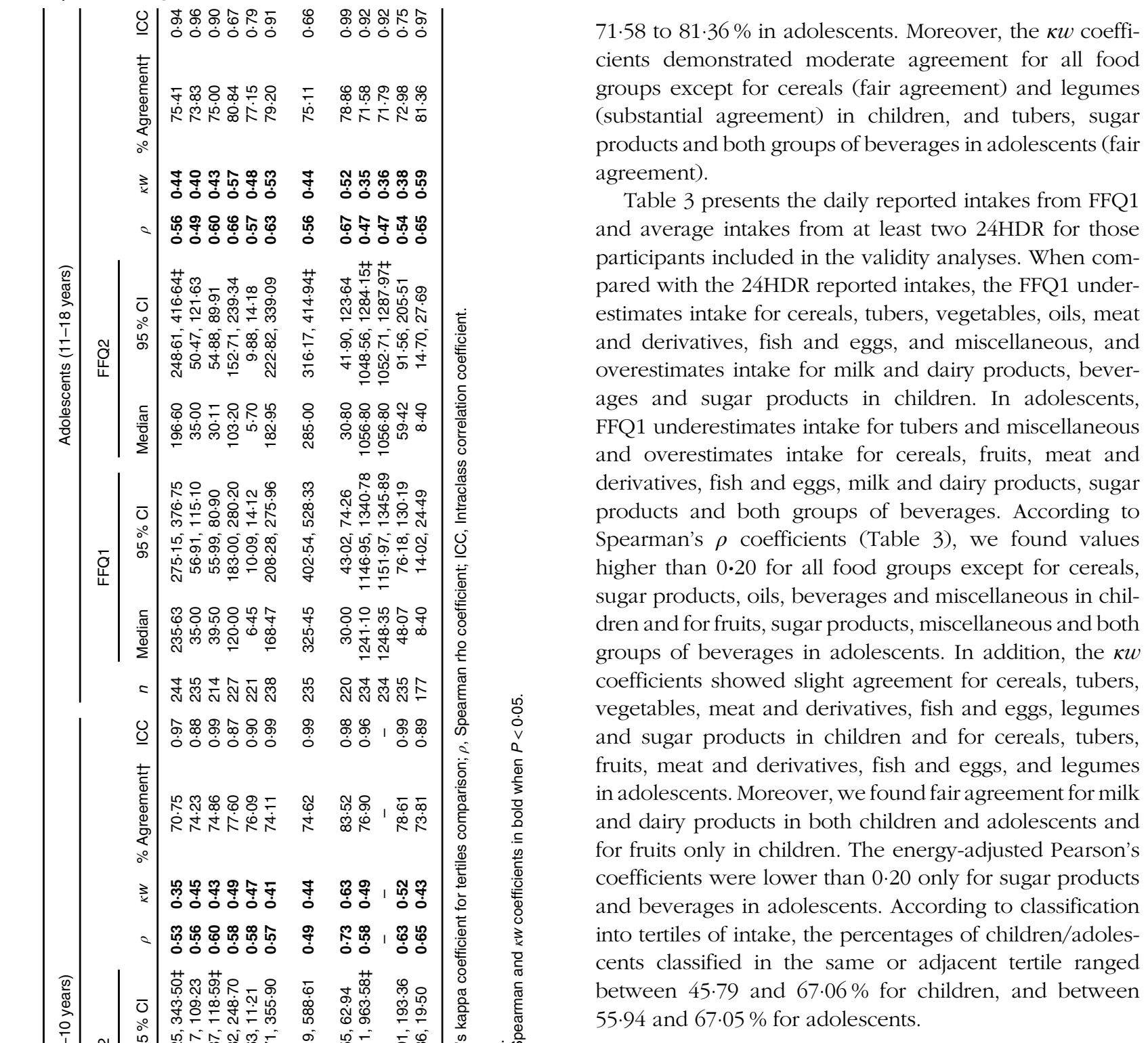

71.58 to $81.36 \%$ in adolescents. Moreover, the $\kappa w$ coefficients demonstrated moderate agreement for all food groups except for cereals (fair agreement) and legumes (substantial agreement) in children, and tubers, sugar products and both groups of beverages in adolescents (fair greement)

Table 3 presents the daily reported intakes from FFQ1 and average intakes from at least two 24HDR for those participants included in the validity analyses. When compared with the 24HDR reported intakes, the FFQ1 underand derivatives, fish and eggs, and miscellaneous, and overestimates intake for milk and dairy products, beverages and sugar products in children. In adolescents, FFQ1 underestimates intake for tubers and miscellaneous and overestimates intake for cereals, fruits, meat and derivatives, fish and eggs, milk and dairy products, sugar products and both groups of beverages. According to Spearman's $\rho$ coefficients (Table 3), we found values higher than 0.20 for all food groups except for cereals, sugar products, oils, beverages and miscellaneous in children and for fruits, sugar products, miscellaneous and both groups of beverages in adolescents. In addition, the $\kappa w$ vegetables, meat and derivatives, fish and eggs, legumes and sugar products in children and for cereals, tubers, fruits, meat and denivatives, fish and eggs, and legunes and dairy products in both children and adolescents and for fruits only in children. The energy-adjusted Pearson's coefficients were lower than $0 \cdot 20$ only for sugar products and beverages in adolescents. According to classification into tertiles of intake, the percentages of children/adolescents classified in the same or adjacent tertile ranged 55.94 and $67.05 \%$ for adolescents.

\section{Discussion}

In the current study, the reliability and validity of a semi-quantitative FFQ was assessed by comparing two administrations of the FFQ over a two-week period and by comparing the FFQ against the $24 \mathrm{HDR}$, respectively. Our results demonstrated a reasonable reliability and slight-to-moderate validity for almost all food groups intakes in children and adolescents.

In the reliability analyses, we found that our FFQ1 overestimated the intake of some food groups when compared to FFQ2. In the literature, some studies reported higher estimates in the first FFQ, and others reported higher estimates in the second $\mathrm{FFQ}^{(21,22)}$. Moreover, our analyses for children and adolescents shows Spearman's correlation coefficients ranging from 0.47 to 0.73 , ICC ranging from 0.66 to 0.99 and $\kappa w$ coefficients ranging from 0.35 to 0.63 . The proportion of participants classified in the same and adjacent 
tertiles, for children and adolescents, varied between 70.75 and $83.52 \%$. In this sense, our FFQ shows an acceptable reliability for both children and adolescents. The correlation and $\kappa w$ coefficients for most food groups were comparable with those reported in other studies in the same population assessing food group reliability ${ }^{(21-25)}$. Moreover, the proportion of participants classified in the same and adjacent tertiles in our study were also similar to that of other studies ${ }^{(21,23,24)}$ in which this proportion is between 70 and $100 \%$.

Our FFQ has a common core of 56 items for all SAYCARE cities, with the addition of typical food items in each centre, which resulted in a list of 65 items on average for each centre ${ }^{(15)}$. Additionally, as previously mentioned, we adopted a 2-week interval for reliability analyses in order to minimize variation in food intake responses due to true changes over time ${ }^{(26)}$. The replication interval should not be too short or too long because in a short span, subjects may remember and repeat answers. On the other hand, a long time interval could be influenced by diet changes, for example changes due to seasonality ${ }^{(27)}$. Although there is no consensus, previous studies have evaluated reliability with the same time interval of 2 weeks for children and adolescents ${ }^{(23,24,26)}$.

In the validation analyses, our FFQ overestimated intake for some food groups and underestimated intake for other groups. As previously reported in other studies ${ }^{(21-23,28)}$, higher intake estimates were reported on FFQ than on 24HDR. For both children and adolescents, our validation analyses demonstrated a Spearman's correlation ranging from 0.17 to $0.37, k w$ coefficients ranging from 0.09 to 0.24 , and proportion of participants classified in the same and adjacent tertile ranging between 45.79 and $67.06 \%$. Although we observed a non-significant $\kappa w$ coefficient or $\kappa w<0 \cdot 20$ (slight agreement) in several food groups, such as oils, beverages, sugar products, fruits or miscellaneous, we found percentages of children/adolescents correctly classified in the same or adjacent tertile ranging from 45.79 to $83.52 \%$, similar to other studies ${ }^{(21,23,24,28,29)}$. Moreover, we found an acceptable correlation $(r>0 \cdot 20)^{(2)}$ between FFQ1 and 24HDR in all food groups in children and in almost all food groups in adolescents (except for sugar products and beverages). Numerous studies have evaluated FFQ validity at the food level among children and adolescents, but the results were inconsistent ${ }^{(21,28-30)}$. The lack of agreement between methods is often due to several factors ${ }^{(7,28)}$, such as proxy reporting from parents, nature of dietary habits at the studied age, and lack of a 'perfect' gold standard tool, among others. As previously reported in a systematic review ${ }^{(8)}$, adolescents may forget what they have eaten, may remember other items although not consumed within the given time span, or some foods may not be recognized because they are part of a dish. Moreover, as mentioned previously, adolescents have difficulties in estimating portion sizes ${ }^{(31,32)}$, which may lead to over- or under-estimation of intake. In addition, parents/ 
guardians' educational level or social desirability could influence reporting, and parents/guardians are unreliable reporters of their children's food intake when out of the home $^{(7)}$, leading to generally lower validity correlations ${ }^{(9)}$. Among South American children, it is common to have not only lunch but also breakfast or morning/afternoon snacks at school. In addition, correlation coefficients may be affected by the manner in which the questionnaire is administered, and the age, sex and ethnicity of population $^{(24)}$. Other factors affecting reported intakes and complicating the comparison among validation studies are the differences in the way the FFQ was developed, design characteristics such as number of items, inclusion of portion sizes or sample size, food groups examined, unit of estimation or statistical method used, use of a reference method and number of recorded days ${ }^{(28,33)}$. Although some authors suggest that the number of items included does not have an impact on validity ${ }^{(34)}$, in a review it was suggested that an extensive list can lead to inaccuracy due to fatigue when filling out the questionnaire ${ }^{(9)}$. In this review, they found that studies in children and adolescents with the strongest validity correlations used medium length questionnaires, ranging from 19 to 63 items $^{(9)}$.

Our study has some limitations. We used a convenience sample of children and adolescents ${ }^{(14)}$, although we included both private and public schools. Moreover, we used $24 \mathrm{HDR}$ as the reference method. Although $24 \mathrm{HDR}$ have acceptable validity in children and adolescents $^{(2-4)}$ and are widely used as a gold stan$\operatorname{dard}^{(8,9,23,35)}$, they are not a perfect measure of dietary intake due to the high degree of intrapersonal variability and the fact that misreporting of energy intake is a common problem, especially in adolescents, which can affect validity results ${ }^{(28,36)}$. In order to mitigate this, in our study, trained dietitians assisted the participants. Preferably, biochemical markers, such as doubly labelled water ${ }^{(37)}$ or several dietary biomarkers ${ }^{(21)}$, should be implemented in validation studies as they can be alternative reference methods to objectively assess energy and dietary consumption because their measurement errors are independent of those from $\mathrm{FFQ}^{(21,37)}$. However, although biochemical markers are valuable as reference methods as they are not affected by errors in recall or other issues, they are not available for all nutrients or for all food groups ${ }^{(2)}$. Moreover, these methods are expensive, require sophisticated laboratories and equipment and do not provide information on all food groups ${ }^{(35)}$. Finally, the time span evaluated in our FFQ was 3 months ${ }^{(15)}$. In this sense, a review found that studies with shorter time span assessment periods (previous day or week) have better validity than those with longer periods (from one month to one year) ${ }^{(9)}$.

On the other hand, the current study has several strengths. First, we followed standardized and harmonized procedures during data collection of the SAYCARE study fieldwork $^{(14)}$. Moreover, we assessed a large enough sample of children and adolescents from seven South American cities and, according to Willett ${ }^{(2)}$, our sample size was optimal for validation studies. Second, our FFQ was developed specifically for children and adolescents. Both the FFQ and 24HDR covered the same time frame, and we developed a food photo booklet ${ }^{(15)}$ to facilitate accurate recordings. In some centres, as children have lunch or other meals at school, schoolteachers helped provide answers when necessary. Third, several studies in this field measured the reliability and validity of FFQ for children and adolescents in terms of nutrients intake. From our point of view, and in accordance with some intervention trials ${ }^{(38)}$, whole foods rather than individual nutrients may best indicate the potential role of diet in disease prevention. Therefore, with the overconsumption of specific food groups associated with diseases (such as obesity in youths), assessing foods or food group intakes with validated methods is especially important ${ }^{(9)}$. In our study, participants answered the FFQ twice with a two-week interval between assessments, and they completed at least two 24HDR. The long recording periods and the repeated measures of the questionnaires may reduce accuracy due to increasing fatigue and boredom and potential alterations of dietary habits, which can increase the probability of drop-outs ${ }^{(28)}$.

\section{Conclusion}

The SAYCARE food frequency questionnaire possesses satisfactory reliability. In addition, the questionnaire has slight-to-moderate validity for almost all food group intakes in South American children and adolescents. Accordingly, this questionnaire is appropriate for the purpose of ranking the intake of individuals within a population.

\section{Acknowledgements}

Acknowledgements: The authors would like to thank all the participants who took part in the study and to Dr. Alex Jones Flores Cassenote for his participation in the making of the food photo booklet. Financial support: Tara RendoUrteaga is in receipt of a post-doctoral scholarship from São Paulo Research Foundation - FAPESP (proc. 2014/ 25233-0). Luisa Saravia is in receipt of a doctoral scholarship from Carolina Foundation. Tatiana Sadalla Collese is in receipt of a doctoral scholarship from FAPESP (proc. 2016/13922-1). Augusto César F. de Moraes is in receipt of a post-doctoral scholarship from National Counsel of Technological and Scientific Development - CNPq (proc. 313772/2014-2) and FAPESP (proc. 2014/13367-2 and 2015/14319-4). Full Professor Luis A. Moreno was given scholarship of visiting professor from FAPESP (proc. 2015/11406-3). Heráclito B. Carvalho received funding grant from FAPESP (proc. 2014/11468-6). The SAYCARE Study was supported mainly by the Brazilian 
Government from CNPq (proc. 471266/2013-2) and São Paulo State Government from FAPESP (proc. 2014/ 11468-6). The SAYCARE Study has also been co-funded by other agencies in the other countries: (i) Collaborative Projects Fund - Instituto Nacional de Salud del Niño Lima; (ii) Sustainability Strategy 2014-2015 at the University of Antioquia, Medellin, Colombia; (iii) Secretary of University Extension and Student Welfare, University

of Buenos Aires; (iv) European Regional Development Fund (MICINN-FEDER) to GENUD Research Group. Conflict of interest: The authors have nothing to disclose. Authorship: T.R.U., T.S.C., L.S., J.M.M.A., F.T. and B.M.O. were involved in the data collection. T.R.U. was involved in the conducting, analysis and writing of the manuscript. T.S.C. was involved in data interpretation and critically reviewed the manuscript. L.S., J.M.M.A., L.I.G.Z., F.T. and B.M.O. critically reviewed the manuscript. L.I.G.Z., F.L.T.L., L.A.M., A.C.F.M. and H.B.C. were involved in the design and funding and critically reviewed the manuscript. All authors approved the final version. Ethics of buman subject participation: This study was conducted according to the guidelines laid down in the Declaration of Helsinki and all procedures involving human subjects/patients were approved by The Research Ethics Committee of each city involved in the study. Written informed consent was obtained from all participants.

\section{References}

1. Livingstone MB, Robson PJ \& Wallace JM (2004) Issues in dietary intake assessment of children and adolescents. $\mathrm{Br} \mathrm{J}$ Nutr 92, Suppl. 2, S213-S222.

2. Willett W (2012) Nutritional Epidemiology, 3rd ed. New York: Oxford University Press.

3. McPherson RS, Hoelscher DM, Alexander M et al. (2000) Dietary assessment methods among school-aged children: validity and reliability. Prev Med 31, S11-S33.

4. Cade J, Thompson R, Burley V et al. (2002) Development, validation and utilisation of food-frequency questionnaires a review. Public Health Nutr 5, 567-587.

5. Magarey A, Watson J, Golley RK et al. (2011) Assessing dietary intake in children and adolescents: considerations and recommendations for obesity research. Int J Pediatr Obes 6, 2-11.

6. Dietary Guidelines Advisory Committee (2015) Scientific Report of the 2015 Dietary Guidelines Advisory Committee: Advisory Report to the Secretary of Health and Human Services and the Secretary of Agriculture. Washington, DC: U.S. Department of Agriculture, Agricultural Research Service.

7. Livingstone MB \& Robson PJ (2000) Measurement of dietary intake in children. Proc Nutr Soc 59, 279-293.

8. Tabacchi G, Amodio E, Di Pasquale M et al. (2014) Validation and reproducibility of dietary assessment methods in adolescents: a systematic literature review. Public Health Nutr 17, 2700-2714.

9. Kolodziejczyk JK, Merchant G \& Norman GJ (2012) Reliability and validity of child/adolescent food frequency questionnaires that assess foods and/or food groups. J Pediatr Gastroenterol Nutr 55, 4-13.
10. Rockett HR \& Colditz GA (1997) Assessing diets of children and adolescents. Am J Clin Nutr 65, 1116S-1122S.

11. Pigeot I, Baranowski T \& De Henauw S (2015) The IDEFICS intervention trial to prevent childhood obesity: design and study methods. Obes Rev 16, Suppl. 2, 4-15.

12. Moreno LA, De Henauw S, Gonzalez-Gross M et al. (2008) Design and implementation of the Healthy Lifestyle in Europe by Nutrition in Adolescence Cross-Sectional Study. Int J Obes (Lond) 32, Suppl. 5, S4-11.

13. Manios Y, Androutsos O, Katsarou C et al. (2014) Designing and implementing a kindergarten-based, family-involved intervention to prevent obesity in early childhood: the ToyBox-study. Obes Rev 15, Suppl. 3, 5-13.

14. Carvalho HB, Moreno LA, Silva AM et al. (2018) Design and Objectives of the South American Youth/Child Cardiovascular and Environmental (SAYCARE) Study. Obesity (Silver Spring) 26, Suppl. 1, S5-S13.

15. Saravia L, Gonzalez-Zapata LI, Rendo-Urteaga T et al. (2018) Development of a food frequency questionnaire for assessing dietary intake in children and adolescents in South America. Obesity (Silver Spring) 26, Suppl. 1, S31-S40.

16. United States Department of Agriculture, Agricultural Research Service USDA (2017) Food Composition Databases. https://ndb.nal.usda.gov/ndb/ (accessed March 2017).

17. International Network of Food Data Systems (INFOODS) Food and Agriculture Organization of the United Nations (2017) Latin American Food Composition Tables. http://www.fao. org/infoods/infoods/tablas-y-bases-de-datos/america-latina/ es/ (accessed March 2017).

18. Food and Agriculture Organization of the United Nations \& World Health Organization (2006) Joint FAO/WHO Food Standards Programme. Codex Classification of Foods and Animal Feeds. Draft Revision-1, pp. 4-9. Rome: Codex Alimentarius Commission.

19. Willett WC, Sampson L, Stampfer MJ et al. (1985) Reproducibility and validity of a semiquantitative food frequency questionnaire. Am J Epidemiol 122, 51-65.

20. Landis JR \& Koch GG (1977) The measurement of observer agreement for categorical data. Biometrics 33, 159-174.

21. Vioque J, Gimenez-Monzo D, Navarrete-Munoz EM et al. (2016) Reproducibility and validity of a food frequency questionnaire designed to assess diet in children aged 4-5 years. PLoS One 11, e0167338.

22. Lanfer A, Hebestreit A, Ahrens W et al. (2011) Reproducibility of food consumption frequencies derived from the Children's Eating Habits Questionnaire used in the IDEFICS study Int J Obes (Lond) 35, Suppl. 1, S61-S68.

23. Vereecken CA \& Maes L (2003) A Belgian study on the reliability and relative validity of the Health Behaviour in SchoolAged Children food-frequency questionnaire. Public Health Nutr 6, 581-588.

24. Saeedi P, Skeaff SA, Wong JE et al. (2016) Reproducibility and relative validity of a short food frequency questionnaire in 9-10 year-old children. Nutrients $\mathbf{8}$, E721.

25. Rockett HR, Wolf AM \& Colditz GA (1995) Development and reproducibility of a food frequency questionnaire to assess diets of older children and adolescents. J Am Diet Assoc 95, 336-340.

26. Metcalf PA, Scragg RKR, Sharpe S et al. (2003) Short-term repeatability of a food frequency questionnaire in New Zealand children aged 1-14 y. Eur J Clin Nutr 57, 1498-1503.

27. Marchioni DML, Voci SM, de Lima FEL et al. (2007) Reproducibility of a food frequency questionnaire for adolescents. Cad Saude Publica 23, 2187-2196.

28. Bel-Serrat S, Mouratidou T, Pala V et al. (2014) Relative validity of the Children's Eating Habits Questionnaire-food frequency section among young European children: the IDEFICS Study. Public Health Nutr 17, 266-276. 
29. Truthmann J, Mensink GBM \& Richter A (2011) Relative validation of the KiGGS Food Frequency Questionnaire among adolescents in Germany. Nutr J 10, 133.

30. Blum RE, Wei EK, Rockett HR et al. (1999) Validation of a food frequency questionnaire in Native American and Caucasian children 1 to 5 years of age. Matern Child Health J 3, 167-172.

31. Cullen KW, Watson K \& Zakeri I (2008) Relative reliability and validity of the Block Kids Questionnaire among youth aged 10 to 17 years. J Am Diet Assoc 108, 862-866.

32. Poslusna K, Ruprich J, de Vries JHM et al. (2009) Misreporting of energy and micronutrient intake estimated by food records and 24 hour recalls, control and adjustment methods in practice. Br J Nutr 101, S73-S85.

33. Molag ML, de Vries JHM, Ocke MC et al. (2007) Design characteristics of food frequency questionnaires in relation to their validity. Am J Epidemiol 166, 1468-1478.
34. Cade JE, Burley VJ, Warm DL et al. (2004) Food-frequency questionnaires: a review of their design, validation and utilisation. Nutr Res Rev 17, 5-22.

35. Rockett HR, Breitenbach M, Frazier AL et al. (1997) Validation of a youth/adolescent food frequency questionnaire. Prev Med 26, 808-816.

36. Block G \& Hartman AM (1989) Issues in reproducibility and validity of dietary studies. Am J Clin Nutr 50, 1133-1135.

37. Bornhorst C, Bel-Serrat S, Pigeot I et al. (2014) Validity of 24-h recalls in (pre-)school aged children: comparison of proxyreported energy intakes with measured energy expenditure. Clin Nutr 33, 79-84.

38. Neuhouser ML, Patterson RE, Thornquist MD et al. (2003) Fruits and vegetables are associated with lower lung cancer risk only in the placebo arm of the beta-carotene and retinol efficacy trial (CARET). Cancer Epidemiol Biomarkers Prev 12, 350-358. 